\title{
Elevated Small Dense Low-Density Lipoprotein Cholesterol as a Predictor for Future Cardiovascular Events in Patients with Stable Coronary Artery Disease
}

\author{
Tenjin Nishikura ${ }^{1}$, Shinji Koba ${ }^{1}$, Yuya Yokota ${ }^{1}$, Tsutomu Hirano ${ }^{2}$, Fumiyoshi Tsunoda ${ }^{1}$, Makoto Shoji ${ }^{1}$, \\ Yuji Hamazaki ${ }^{1}$, Hiroshi Suzuki ${ }^{3}$, Yasuki Itoh ${ }^{4}$, Takashi Katagiri ${ }^{1}$ and Youichi Kobayashi ${ }^{1}$ \\ ${ }^{1}$ The Department of Medicine, Division of Cardiology, Showa University School of Medicine, Tokyo, Japan \\ ${ }^{2}$ The Department of Medicine, Division of Diabetes, Metabolism and Endocrinology, Showa University School of Medicine, Tokyo, \\ Japan \\ ${ }^{3}$ The Department of Medicine, Division of Cardiology Showa University Fujigaoka Hospital, Yokohama City, Kanagawa, Japan \\ ${ }^{4}$ Research and Development Department, Denka Seiken Co. Ltd, Tokyo, Japan
}

\begin{abstract}
Aim: The aim of the present study was to investigate how small dense low-density lipoprotein cholesterol (sdLDL-C) compared with LDL-C affect the long-term prognosis in patients with stable coronary artery disease $(\mathrm{CAD})$.

Methods: sdLDL-C measured by heparin magnesium precipitation and LDL particle size measured by non-denatured gradient-gel electrophoresis were compared in 190 consecutive CAD patients who underwent coronary arteriography between 2003 and 2004 who did or did not develop cardiovascular events during a seven-year follow-up period. Cardiovascular events were death caused by cardiovascular diseases (CVDs), onset of acute coronary syndrome, need for coronary and peripheral arterial revascularization, hospitalization for heart failure, surgical procedure for any CVDs, and/or hospitalization for stroke.

Results: First-time cardiovascular events were observed in 72 patients. Those who experienced cardiovascular events were older and had higher prevalence rates of hypertension and diabetes; significantly higher Gensini coronary atherosclerotic scores; significantly higher levels of sdLDL-C, sdLDL-C/LDL$\mathrm{C}$, and LDL-C/high-density lipoprotein cholesterol (HDL-C) ratios; and greater glycated hemoglobin $(\mathrm{Hb}) \mathrm{A} 1 \mathrm{c}$ and brain natriuretic peptide (BNP) levels. They also had significantly smaller LDL particle sizes, HDL-C, apolipoprotein A-1, and estimated glomerular filtration rate (GFR) compared with patients without cardiovascular events. Conversely, LDL-C, non-HDL-C, apolipoprotein B, remnantlike particle cholesterol, and high-sensitivity C-reactive protein (hs-CRP) levels were similar between the two groups. A Kaplan-Meyer event-free survival curve demonstrated that patients with sdLDL-C $\geq 35 \mathrm{mg} / \mathrm{dL}$ (median level) had significantly poorer prognosis compared with those with lower sdLDL$\mathrm{C}$ levels, while patients with LDL-C $\geq 100 \mathrm{mg} / \mathrm{dL}$ had a non-significantly lower survival rate.

Conclusion: These results confirm that sdLDL-C is a very promising biomarker to predict future cardiovascular events in the secondary prevention of stable CAD.
\end{abstract}

J Atheroscler Thromb, 2014; 21:755-767.

Key words: Small dense LDL, Lipids, Cardiovascular disease, Coronary artery disease, Secondary prevention

Address for correspondence: Shinji Koba, Department of Medicine, Division of Cardiology, Showa University School of Medicine, 1-5-8 Hatanodai, Shinagawa-ku, Tokyo 1428666, Japan

E-mail: skoba@med.showa-u.ac.jp

Received: December 28, 2013

Accepted for publication: January 29, 2014

\section{Introduction}

Low-density lipoprotein cholesterol (LDL-C) level has been the cornerstone of lipid diagnosis and therapy ${ }^{1)}$. LDL particles are fractionated into large buoyant and small dense particles ${ }^{2)}$. Small dense LDL (sdLDL) particles have been suggested to be more ath- 
erogenic than large buoyant LDL due to several characteristics, including higher penetration into the arterial wall, lower binding affinity for the LDL receptor, prolonged plasma half-life, and lower resistance to oxidative stress ${ }^{2-4)}$. Several methods have been developed for the measurement of serum sdLDL levels, 5 ).

Cross-sectional studies, including ours, have used gradient gel electrophoresis to demonstrate that the predominance of sdLDL is associated with a three-fold increased risk for coronary artery disease $(\mathrm{CAD})^{7,8)}$. The prospective Québec cardiovascular study confirmed that a greater proportion of sdLDL evaluated by semiquantitative estimation using gradient gel electrophoresis at baseline is a strong and independent predictor of CAD in the first seven years of follow-up ${ }^{9)}$. In contrast, an elevated concentration of large LDL is a poor predictor of CAD and seems to be associated with a low CAD risk ${ }^{9}$. In earlier cross-sectional studies using the heparin magnesium precipitation method for quantification of sdLDL cholesterol (sdLDL-C), we demonstrated that a high sdLDL-C concentration, but not large LDL-C level, is closely related to the angiographic and/or clinical severity of CAD independent of classical coronary risk factors ${ }^{10,11)}$. Similarly, a case-control analysis from the Framingham Offspring study using the same method of sdLDL-C measurement showed that the sdLDL-C/LDL-C ratio was significantly higher in subjects with CAD than in those without CAD regardless of gender, whereas LDL-C and large LDL-C are somewhat lower in those patients with $\mathrm{CAD}^{12}$. The Suita study, which assessed a prospective cohort study of an Japanese urban general population, reported that increases in sdLDL-C, as measured by another direct homogenous assay of sdLDL-C, were significantly associated with the onset of cardiovascular disease (CVD), especially myocardial infarction (MI), independently of LDL-C ${ }^{13)}$. On the basis of these studies, sdLDL particles seem likely to be major atherogenic components of LDL particles.

However, no prospective study has evaluated the relative strengths of the associations of sdLDL-C and LDL-C with cardiovascular event recurrence among patients with CAD. In addition, no prospective study has compared sdLDL-C with other useful lipid biomarkers, such as non high-density lipoprotein (HDL)cholesterol (non-HDL-C), apolipoprotein B, lipoprotein(a), remnant lipoprotein cholesterol (RLP$\mathrm{C})$, HDL-C, and the LDL-C/HDL-C ratio ${ }^{14,15)}$. The aim of the present study is to determine whether sdLDL-C is superior to LDL-C and/or other lipid biomarkers for predicting future cardiovascular events in patients with stable CAD.

\section{Methods}

\section{Subjects}

This observational study cohort consisted of 258 male and 70 female consecutive patients with angiographically documented CAD who underwent diagnostic coronary arteriography and measurement of sdLDL-C and LDL particle diameter (LDL-PD) at Showa University Hospital between September 2003 and December 2004. Data from repeated angiograms for the included patients, patients who underwent emergency coronary angiography due to acute coronary syndrome (ACS), and patients who underwent hemodialysis therapy were not included. At the time the measurements were made, aggressive lipid-lowering treatment was not common in patients with CAD, and drug-eluting stents were not routinely used. Significant $\mathrm{CAD}$ was defined as a $50 \%$ or greater narrowing of the diameter in one or more coronary artery branch on an arteriogram. The diagnoses of CAD, such as MI and angina pectoris, were based on clinical symptoms, electrocardiographic changes, myocardial perfusion scintigraphy, blood examinations, coronary arteriograms, and a prior history of percutaneous coronary intervention (PCI) and/or coronary artery bypass surgery $(\mathrm{CABG})$. In the present analysis, the exclusion criteria were age of 90 years or older, severe hepatic disease, nephrotic syndrome or renal dysfunction (serum creatinine $>1.5 \mathrm{mg} / \mathrm{dL}$ ), infectious disease, current treatment for malignancy, any other serious condition, patients who took drugs for thyroid dysfunction, or patients with missing blood examination data. Patients who could not be followed within two months after the coronary angiography were also excluded. After applying these criteria, data from 149 men and 41 women were included in the present analysis. The institutional review board of Showa University approved this protocol. The investigation conformed to the principles of the Declaration of Helsinki.

\section{Baseline Examination}

The fasting blood samples were obtained by venipuncture immediate before the cardiac catheterization study was performed. The serum was stored at $4{ }^{\circ} \mathrm{C}$ and assayed within three days after sampling. The CAD group consisted of patients with previous MI (87 men and 19 women) and stable angina pectoris (92 men and 26 women). Ninety-three men and 20 women had previously undergone PCI. Five men and one woman had previously received CABG. Sixtyeight men and 26 women were taking lipid-lowering drugs at baseline. Seven men and two women were 
previously treated for malignancy. Eighty men and 17 women underwent coronary revascularization due to coronary angiography findings at baseline.

The diagnosis of hypertension was based on a history of hypertension or blood pressure above 140 $\mathrm{mmHg}$ systolic or $90 \mathrm{mmHg}$ diastolic. Diabetes mellitus was defined as a fasting serum glucose value greater than $126 \mathrm{mg} / \mathrm{dL}$, glycated hemoglobin $(\mathrm{Hb})$ A1c values estimated by $(=1.019 \times \mathrm{HbA1c}$ [Japan Diabetes Society, JDS] + 0.3) greater than 6.5\%, and/ or the current use of medication for diabetes ${ }^{16}$. Dyslipidemia was defined as the current use of lipid-lowering medication and/or meeting the criteria of the Japan Atherosclerosis Society for fasting serum lipid levels as follows LDL-C $\geq 140 \mathrm{mg} / \mathrm{dL}$, HDL-C $<40$ $\mathrm{mg} / \mathrm{dL}$, or $\mathrm{TG} \geq 150 \mathrm{mg} / \mathrm{dL}^{17)}$. A serum creatininebased estimate of glomerular filtration rate (eGFR) was calculated ${ }^{18)}$. Body mass index (BMI) was calculated as weight $(\mathrm{kg})$ divided by height $(\mathrm{m})$ squared. Patients with a reported smoking habit of at least one cigarette per day on admission were classified as current smokers.

\section{Coronary Atherosclerosis Severity and Definition of Major Cardiovascular Events}

The severity of coronary atherosclerosis was estimated by calculating the Gensini score, an established method for grading CAD severity ${ }^{19)}$ and by quantifying the number of significantly stenotic coronary vessels, which were defined as more than 50\% narrowing of the diameter in the left main coronary artery or more than $75 \%$ narrowing of the diameter in one or more branches of the other coronary arteries.

In-hospital medical records were evaluated between August and September 2010. The endpoints were: (1) the date of the first cardiovascular event onset, (2) the date of death, and (3) the date of the patient's last visit to Showa University Hospital. Cardiovascular events were defined as death caused by CVDs; onset of ACS; need for coronary or any peripheral arterial revascularization including restenosis after PCI; heart failure hospitalization; surgical procedure for any CVDs; and/or requiring hospitalization for ischemic or hemorrhagic stroke. Coronary revascularization within two months was not identified as a cardiovascular event because it was indistinguishable from unexpected or scheduled coronary revascularization.

\section{Lipoprotein Levels, Inflammatory Parameters, and Brain Natriuretic Peptide Measurements}

Total cholesterol, triglyceride, HDL-C, HbA1c (JDS), and lipoprotein(a) were measured using stan-
Table 1. Cardiovascular events

\begin{tabular}{lc}
\hline \multicolumn{1}{c}{ Cardiovascular events } & $\begin{array}{c}\text { Number } \\
(n=72)\end{array}$ \\
\hline Fatal events & 1 \\
$\quad$ acute coronary syndrome & 9 \\
$\quad$ congestive heart failure & \\
No fatal events & 10 \\
acute coronary syndrome & 14 \\
congestive heart failure & 35 \\
any revascularization & 1 \\
Surgical repair of abdominal aortic aneurysm & 2 \\
Stroke & \\
\hline
\end{tabular}

dard laboratory procedures. Serum apolipoprotein levels were determined by an immunoturbidometric assay (Daiichi Chemicals Co., Tokyo, Japan). RLPs were isolated from the serum to an immunoaffinity mixed gel containing anti-apolipoprotein $\mathrm{A} 1$ and anti-apolipoprotein B100 monoclonal antibodies (Japan Immunoresearch Laboratories, Takasaki, Japan), and the cholesterol concentrations of the unbound fraction were measured as RLP-C ${ }^{20)}$. The LDL-C levels were measured with a direct homogenous assay of the serum using detergents (LDL-EX, Denka Seiken, Tokyo, Japan ${ }^{21)}$. sdLDL-C levels were measured with a rapid assay using a modified version of a previously described heparin-magnesium precipitation method ${ }^{22,23)}$. The kits used for the LDL-C and sdLDL-C tests were both provided by Denka Seiken. Because we found a strong correlation between cholesterol concentrations and apolipoprotein B concentrations in the sdLDL fraction $(r=0.944, p<0.0001)$ and strong correlations of cholesterol and apolipoprotein B concentrations between the sdLDL fraction by this precipitation method and the dense LDL fraction (density $=1.044$ $1.063)$ by ultracentrifugation $(r=0.884, p<0.0001$ and $r=0.896, p<0.0001$, respectively), we conjectured that sdLDL-C measurement would be sufficient to evaluate the sdLDL particle number, that is, the sdLDL mass ${ }^{23}$. Large LDL-C was estimated by subtracting the sdLDL-C concentration from the LDL-C concentration, and the sdLDL-C to LDL-C ratio was also calculated from direct measurements. Our group has previously shown that the large LDL-C values estimated by this method are correlated with actual values determined by ultracentrifugation $(r=0.858, p<$ $0.0001)^{23)}$. Non-HDL-C was estimated by subtracting the HDL-C concentration from the total-cholesterol concentration ${ }^{17)}$. The peak LDL particle diameter was determined by $2-16 \%$ non-denatured polyacrylamide gel electrophoresis according to the method of Nich- 
Table 2. Clinical characteristics at baseline

\begin{tabular}{|c|c|c|c|c|}
\hline variable & $\begin{array}{c}\text { Whole } \\
(n=190)\end{array}$ & $\begin{array}{c}\text { Event } \\
(n=72)\end{array}$ & $\begin{array}{l}\text { Non-event } \\
(n=118)\end{array}$ & $p$ \\
\hline Gender, men/women & $149 / 41$ & $60 / 12$ & $89 / 29$ & 0.134 \\
\hline Age, years & $65.5 \pm 11.5$ & $68.7 \pm 10.9$ & $63.5 \pm 11.4$ & 0.003 \\
\hline Body mass index, $\mathrm{kg} / \mathrm{m}^{2}$ & $24.2 \pm 3.3$ & $24.6 \pm 3.4$ & $23.4 \pm 3.3$ & 0.222 \\
\hline \multicolumn{5}{|l|}{ Diagnosis } \\
\hline Angina pectoris & $118(62.1 \%)$ & $46(63.9 \%)$ & $72(61.0 \%)$ & \multirow{3}{*}{0.131} \\
\hline Previous myocardial infarction & $72(37.9 \%)$ & $26(36.1 \%)$ & $46(39.0 \%)$ & \\
\hline Prior history of PCI/CABG & $110 / 6$ & $38 / 3$ & $72 / 3$ & \\
\hline \multicolumn{5}{|l|}{ Coronary angiography } \\
\hline Stenotic vessel number $0 / 1 / 2 / 3$ & $25 / 47 / 53 / 65$ & $2 / 13 / 19 / 38$ & $23 / 34 / 34 / 27$ & $<0.0001$ \\
\hline Gensini Score & $42.0 \pm 39.6$ & $64.4 \pm 43.0$ & $28.2 \pm 30.1$ & $<0.0001$ \\
\hline Need for coronary revascularization & $97(51.1 \%)$ & $51(70.1 \%)$ & $46(39.0 \%)$ & $<0.0001$ \\
\hline \multicolumn{5}{|l|}{ Risk factors } \\
\hline Hypertension & $152(80 \%)$ & $64(88.9 \%)$ & $88(74.6 \%)$ & 0.012 \\
\hline Diabetes mellitus & $86(45.3 \%)$ & $44(61.1 \%)$ & $42(35.6 \%)$ & 0.001 \\
\hline Dyslipidemia & $162(85.3 \%)$ & $62(86.1 \%)$ & $100(84.7 \%)$ & 0.486 \\
\hline \multicolumn{5}{|l|}{ Smoking } \\
\hline Current & $49(25.8 \%)$ & $18(25.0 \%)$ & $31(26.3 \%)$ & \multirow{2}{*}{0.575} \\
\hline Former & $66(34.7 \%)$ & $28(38.9 \%)$ & $38(52.8 \%)$ & \\
\hline Family history & $35(18.4 \%)$ & $16(22.2 \%)$ & $19(16.1 \%)$ & 0.136 \\
\hline History of Malignancy & $9(4.7 \%)$ & $4(5.6 \%)$ & $5(4.2 \%)$ & 0.464 \\
\hline \multicolumn{5}{|l|}{ Medication } \\
\hline Calcium Channel Blocker & $86(45.3 \%)$ & $39(54.2 \%)$ & $47(39.8 \%)$ & 0.031 \\
\hline Angiotensin type 1 receptor blocker & $81(42.6 \%)$ & $35(48.6 \%)$ & $46(49.0 \%)$ & 0.033 \\
\hline Angiotensin converting enzyme inhibitors & $36(18.9 \%)$ & $19(26.4 \%)$ & $17(14.4 \%)$ & 0.125 \\
\hline Beta-blocker & $43(22.6 \%)$ & $19(26.4 \%)$ & $24(20.3 \%)$ & 0.215 \\
\hline Antiplatelet & $147(77.4 \%)$ & $54(25.0 \%)$ & $93(78.8 \%)$ & 0.331 \\
\hline Oral anti-diabetic drugs & $64(33.7 \%)$ & $31(43.1 \%)$ & $33(28.0 \%)$ & \multirow{2}{*}{0.002} \\
\hline Insulin therapy & $13(6.8 \%)$ & $9(12.5 \%)$ & $4(3.4 \%)$ & \\
\hline Lipid-lowering & $94(49.5 \%)$ & $34(47.2 \%)$ & $60(50.8 \%)$ & \multirow{4}{*}{0.359} \\
\hline Statin & $82(43.2 \%)$ & $27(37.5 \%)$ & $55(46.6 \%)$ & \\
\hline Fibrate & $6(3.2 \%)$ & $4(5.6 \%)$ & $2(1.7 \%)$ & \\
\hline Others except statin and fibrate & $11(5.8 \%)$ & $5(6.9 \%)$ & $6(5.1 \%)$ & \\
\hline
\end{tabular}

Data are expressed as the mean \pm SD, or number (\%)

ols, et al. ${ }^{24)}$. The sdLDL phenotype was defined as a diameter equal to or less than $25.5 \mathrm{~nm}^{2)}$. The highsensitivity C-reactive protein (hs-CRP) level was measured by the Dade Behring BN assay ${ }^{25)}$. Plasma brain natriuretic peptide (BNP) was measured by radioimmunoassays.

\section{Statistics}

All statistical analyses were performed using the SPSS 18.0 software package (SAS Institute, Cary, NC, USA). Baseline characteristics were compared between patients who developed first-time cardiovascular events during follow-up versus those who did not using analyses of variance (ANOVAs) for parametric variables and Wilcoxon tests for non-parametric variables. All variables except eGFR failed to demonstrate a Gaussian distribution. Categorical variables were compared with chi-square tests. Correlation coefficients between LDL-C, sdLDL-C or large LDL-C, and various parameters were determined by Spearman's rank analyses. Among three groups based on the presence or absence of lipid-lowering medication at baseline and during follow-up, sdLDL-C, LDL-C, and HDL-C levels at baseline and at the last visit were compared using the Wilcoxon test, and changes in LDL-C and HDL-C were compared at four time points (baseline, one year, three \pm one year, and six years) using repeated-measures ANOVA among living 
Table 3. Laboratory characteristics at baseline

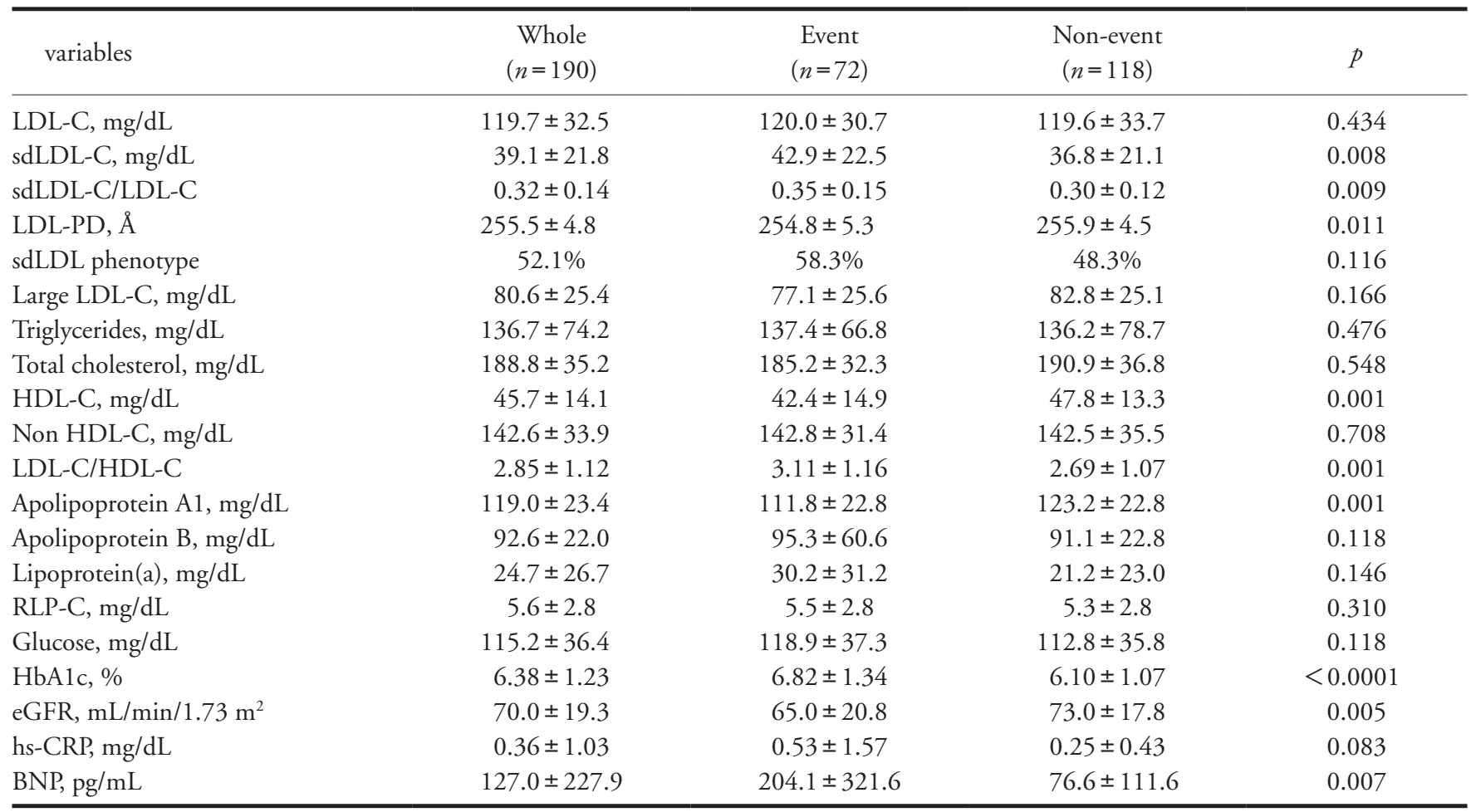

Data are expressed as the mean $\pm \mathrm{SD}$, or $\%$

patients without missing data. Although this was a case-control study, cumulative incidence was estimated by the Kaplan-Meier method in patients divided into two groups based on whether they were above or below the median levels for sdLDL-C and target levels of LDL-C and HDL-C according to the Japan Atherosclerosis Society ${ }^{17)}$ and had an LDL-C/ HDL-C ratio smaller or larger than 2.0, which has been reported to be associated with coronary plaque progression according to a recent meta-analysis using intravascular ultrasound ${ }^{15)}$; differences were assessed with log-rank tests. The day of the baseline measurement of lipids was set as the landmark point, and cardiovascular outcomes were evaluated from this point on. Clinical data on patients who moved during follow-up were identified as censored cases and were used for the period for which they could be followed. Cox regression analysis was used to calculate hazard ratios (HRs) and corresponding 95\% confidence intervals (CIs) for the risk of future cardiovascular events, adjusting for differences in factors that differed significantly between patients with and without cardiovascular events. All the statistical analyses were two-tailed. $P<0.05$ was considered statistically significant.

\section{Results}

Baseline Characteristics and Cardiovascular Events The median follow-up period was 5.8 years. The complete seven-year follow-up rate was $58 \%$ while complete one-year, three-year and five-year follow-up rates were $99 \%, 87 \%$, and $74 \%$, in respectively. Ten patients died of CVDs (one acute MI and nine heart failure) and 15 patients died of non-CVD. Table 1 shows the cardiovascular events. First-time cardiovascular events were observed in 72 patients. Eleven patients were admitted due to ACS, 23 patients were hospitalized due to congestive heart failure, 35 patients underwent revascularization, one patient received surgical treatment for abdominal aortic aneurysm, and two patients were admitted due to ischemic stroke. No patients were admitted due to hemorrhagic stroke. Table 2 compares the general characteristics between the patients with and without cardiovascular events. The patients with cardiovascular events were older; had significantly higher prevalence rates of hypertension and diabetes; were more likely to be under insulin therapy; and had higher Gensini Scores, multi-vessel disease, and need for coronary revascularization at baseline compared with event-free patients. Table 3 compares the laboratory findings between patients 
Table 4. Spearman's correlation between LDL-C, sdLDL-C, and large LDL-C and clinical and lipid parameters

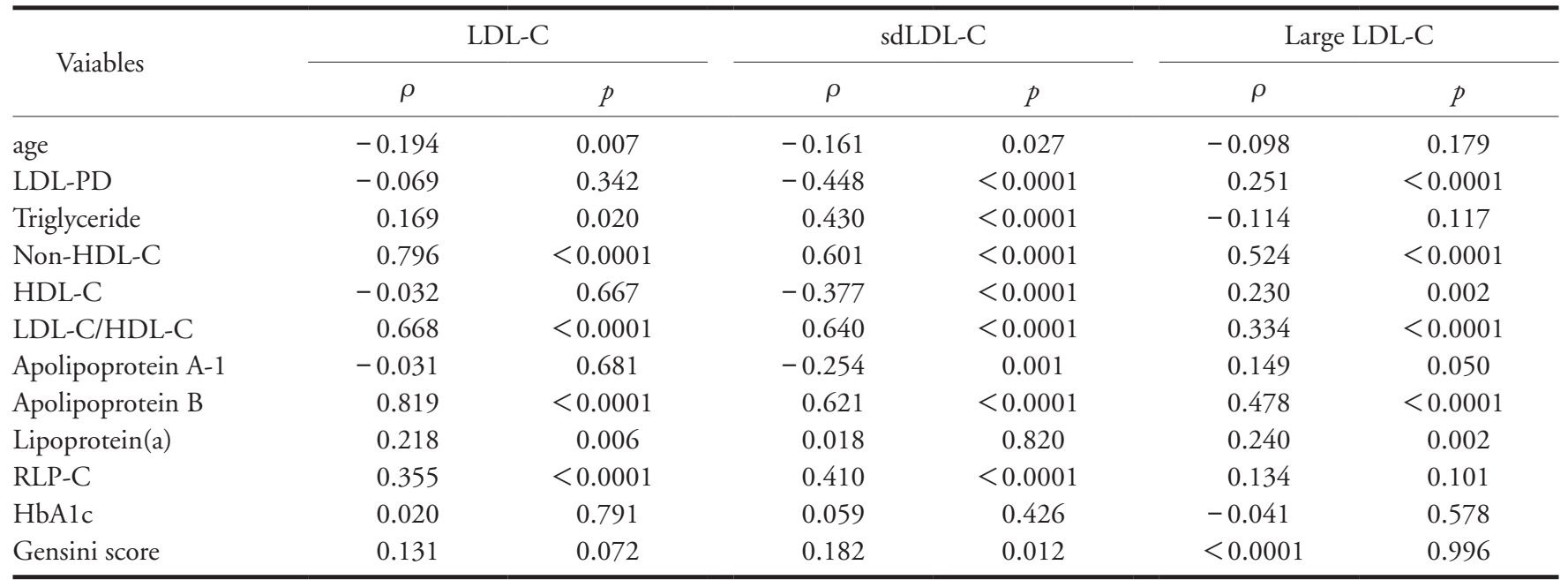

Spearman's correlation are expressed as $\rho$.

with and without cardiovascular events. The patients with cardiovascular events had significantly higher levels of sdLDL-C, sdLDL-C/LDL-C, LDL-C/HDL-C, $\mathrm{HbA1c}$, and BNP and significantly lower levels of LDL-PD, HDL-C and apolipoprotein A-1 compared with those without cardiovascular events. The prevalence of sdLDL phenotype was higher, and hsCRP levels were greater in patients with cardiovascular events; however, the differences were not statistically significant. Conversely, LDL-C, non HDL-C, apolipoprotein B, and RLP-C were similar between the two groups. In addition, total cholesterol and large LDL-C were somewhat lower in the patients who experienced cardiovascular events.

Correlation Coefficients between LDL-C, sdLDL-C, or Large LDL-C and Various Parameters at Baseline

Table 4 lists the correlation coefficients between LDL-C, sdLDL-C, or large LDL-C and various parameters at baseline as evaluated by Spearman's rank analyses. sdLDL-C was significantly positively correlated with triglyceride, non-HDL-C, LDL-C/HDL-C, apolipoprotein B, RLP-C, and Gensini score and was significantly negatively correlated with LDL-PD, HDL$\mathrm{C}$, and apolipoprotein A1. On the other hand, large LDL-C had a weak and somewhat positive correlation with HDL-C and apolipoprotein A1 but was not correlated with triglyceride or RLP-C levels or Gensini score. These results suggest that only sdLDL was significantly associated with atherogenic factors among LDL particles.

\section{Kaplan-Meier Event-Free Survival Analysis and Cox Regression Analysis}

Among 15 patients who died of non-CVD, six patients had non-fatal cardiovascular events, and the others were identified as censored cases. First-time cardiovascular events were observed in 72 patients. The Kaplan-Meier event-free survival curves among patients above or below the median levels for sdLDL$\mathrm{C}$, target levels of LDL-C and HDL-C, and a LDL-C/ HDL-C ratio of 2.0 are illustrated in Fig. 1. The survival curves began to separate in the first few years of follow-up in patients with higher sdLDL-C levels (logrank 4.605, $p<0.05)$. Patients with LDL-C/HDL-C $\geq 2.0$ and those with low HDL-C were also at increased risk for cardiovascular events (log-rank 3.915, $p<$ 0.05 , and 7.307, $p<0.01$, respectively). The survival rate was lower in patients with LDL-C $\geq 100 \mathrm{mg} / \mathrm{dL}$, however, the difference was not statistically significant.

The Cox regression analysis results are shown in Table 5. Increases in sdLDL-C showed a non-significant trend for an increased risk of cardiovascular events, while increases in sdLDL-C/LDL-C ratio showed a 5.78-fold increased risk for cardiovascular events. Age- and gender-matched Cox multivariate regression analysis including sdLDL-C/LDL-C, HDLC, LDL-C/HDL-C, HbAlc, eGFR, hsCRP, BNP, and Gensini score, showed that only Gensini score was significantly associated with cardiovascular events (HR $1.014,95 \%$ CI $1.008-1.020, p<0.0001)$. When the Gensini score was excluded from the Cox multivariate model, HbA1c, hsCRP, and BNP were significantly associated with cardiovascular events; however, sdLDL-C/LDL-C ratio, HDL-C, and LDL-C/HDL-C 


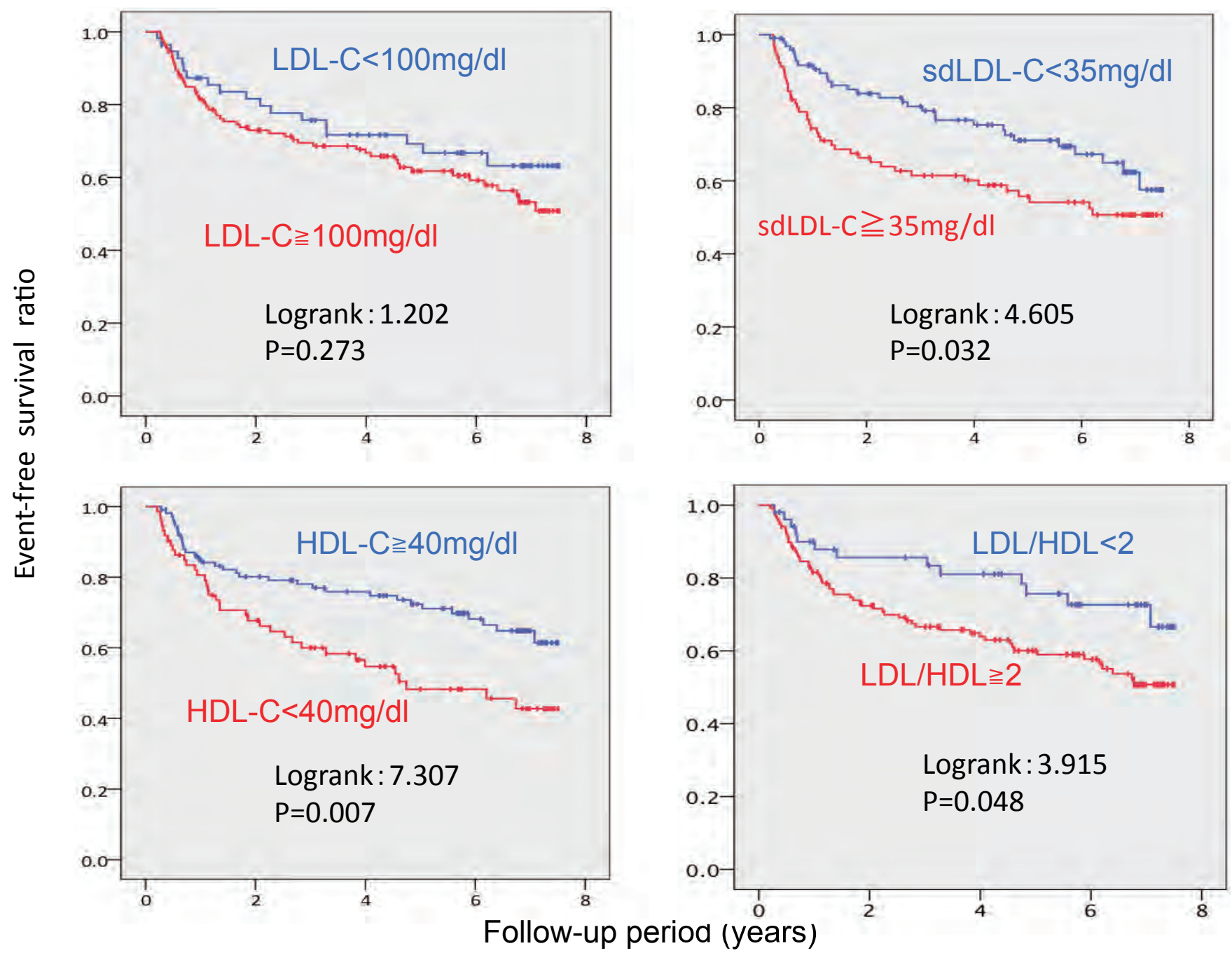

Fig. 1. Kaplan-Meier event-free survival curves. Patients were divided into two groups based on whether their sdLDL-C levels were above or below the median $(35 \mathrm{mg} / \mathrm{dL})$, and target levels of LDL-C (100 mg/dL), HDL-C (40 mg/dL), and the LDL-C/ HDL-C ratio (2.0). The numbers of patients were 134 with LDL-C $\geq 100 \mathrm{mg} / \mathrm{dL}, 56$ with LDL-C $<100 \mathrm{mg} / \mathrm{dL}, 92$ with sdLDL-C $\geq 35 \mathrm{mg} / \mathrm{dL}, 98$ with sdLDL-C $<35 \mathrm{mg} / \mathrm{dL}, 75$ with HDL-C $<40 \mathrm{mg} / \mathrm{dL}, 115$ with HDL-C $\geq 40 \mathrm{mg} / \mathrm{dL}, 51$ with LDL-C/HDL-C $<2.0$, and 139 with LDL-C/HDL-C $\geq 2.0$.

ratio failed to significant associations. These results suggest that poorly controlled diabetes, advanced heart failure, and severe coronary atherosclerosis rather than dyslipidemia might contribute to cardiovascular events.

\section{Sub-Analysis in Patients not Treated with Lipid- Lowering Drugs}

At baseline, 94 patients were treated with lipidlowering drugs, and 30 men and seven women started to take lipid-lowering drugs, especially statin, during the follow-up period. The remaining 51 men and eight women did not take lipid-lowering drugs during the study period. The patients were classified into three groups based on the presence or absence of lipidlowering medication at baseline and during follow-up. Patients who started lipid-lowering drugs during the follow-up period had higher LDL-C and lower HDL-C levels at baseline compared with other groups. All studied patients had high-risk CAD, and they might have changed their lifestyles, resulting in changes in LDL-C and HDL-C levels in the followup period. Indeed, the mean LDL-C and HDL-C levels at their last visits were $101.9 \pm 30.8 \mathrm{mg} / \mathrm{dL}$ and $46.2 \pm 13.3 \mathrm{mg} / \mathrm{dL}$, respectively (Table 6). In addition, the incidences of cardiovascular events were similar among the three groups. Patients who took any 
Table 5. Predictors for cardiovascular events according to Cox’s proportional hazard analysis

\begin{tabular}{|c|c|c|c|c|c|c|c|}
\hline \multirow{3}{*}{ Variable } & \multirow{3}{*}{$\begin{array}{c}\text { Univariate model } \\
\text { HR } 95 \% \text { CI }\end{array}$} & \multicolumn{6}{|c|}{ Multivariate model } \\
\hline & & \multicolumn{2}{|c|}{ Model 1} & \multicolumn{2}{|c|}{ Model 2} & \multicolumn{2}{|c|}{ Model 3} \\
\hline & & HR & $95 \% \mathrm{CI}$ & HR & $95 \% \mathrm{CI}$ & $\mathrm{HR}$ & $95 \% \mathrm{CI}$ \\
\hline age & $1.040(1.016-1.065)^{* * *}$ & 1.032 & $1.000-1.065^{*}$ & 1.045 & $1.018-1.073^{* * *}$ & 1.042 & $1.018-1.067^{* * *}$ \\
\hline men & $0.640(0.344-1.191)$ & 0.757 & $0.392-1.463$ & 0.590 & $0.304-1.146$ & 0.619 & $0.317-1.208$ \\
\hline sdLDL-C/LDL-C & $5.780(1.172-28.503)^{*}$ & 2.368 & $0.345-16.236$ & 2.344 & $0.427-12.867$ & 2.597 & $0.430-15.457$ \\
\hline HDL-C & $0.973(0.952-0.993)^{* *}$ & 0.990 & $0.958-1.023$ & - & - & 0.986 & $0.962-1.009$ \\
\hline Non HDLL-C & $1.001(0.994-1.008)$ & - & - & - & - & - & - \\
\hline LDL-C/HDL-C & $1.298(1.070-1.574)^{* *}$ & 1.130 & $0.842-1.515$ & - & - & - & - \\
\hline Apolipoprotein A1 & $0.982(0.971-0.994)^{* *}$ & - & - & 0.990 & $0.977-1.003$ & - & - \\
\hline Gensini Score & $1.017(1.012-1.022)^{* * *}$ & - & - & - & - & - & - \\
\hline Lipid-lowering medication & $0.818(0.497-1.345)$ & - & - & - & - & - & - \\
\hline
\end{tabular}

Model 1 was adjusted for age, gender, sdLDL-C/LDL-C, HDL-C, LDL-C/HDL-C, HbA1c, eGFR, hs-CRP, BNP. Model 2 was adjusted for age, gender, sdLDL-C/LDL-C, and apolipoprotein A1. Model 3 was adjusted for age, gender, sdLDL-C/LDL-C, and HDL-C.

${ }^{*} p<0.05,{ }^{* *} p<0.01,{ }^{* * *} p<0.001$

lipid-lowering drugs during follow-up and patients with a past history of malignancy were excluded because of effects in sdLDL-C. In the remaining 59 patients, biomarkers were compared among patients with or without cardiovascular events (Table 7). Patients with cardiovascular events had slightly higher sdLDL-C and sdLDL-C/LDL-C levels and significantly higher $\mathrm{HbA1c}$, as well as significantly lower large LDL-C levels than those without cardiovascular events.

\section{Discussion}

To the best of our knowledge, this is the first study to show that elevated sdLDL-C levels compared with LDL-C are strongly associated with cardiovascular event recurrence among patients with stable CAD. Previous cross-sectional studies have demonstrated that patients with CAD showed significantly higher sdLDL-C levels compared with non-CAD controls $\left.{ }^{10}, 11,26,27\right)$. In addition, our previous studies demonstrated that sdLDL-C levels were significantly higher in ACS patients and stable CAD patients with severe coronary atherosclerosis compared with stable CAD patients with mild coronary atherosclerosis. On the other hand, large LDL-C estimated by the subtraction of sdLDL-C from LDL-C were modestly lower in those patients with clinically severe $\mathrm{CAD}^{10,11)}$. These results suggest that atherogenic LDL particles can be attributed to sdLDL; there is an association between high levels of sdLDL-C and severe CAD. However, it is difficult to conclude that elevated sdLDL-C is associated with CAD progression based solely on cross-sectional studies. In prospective cohort studies for primary prevention, elevated levels of sdLDL-C evaluated by different methods have been shown to be associated with an increased risk of CAD onset ${ }^{13)}$. However, it remains to be determined as to whether higher sdLDL-C levels are associated with future cardiovascular events among CAD patients.

The major limitation of the present study is the small sample size and low follow-up rate. However, the follow-up rate at one and three years were $99 \%$ and $87 \%$, respectively; therefore, it is conceivable that cardiovascular events in the short-term could be detected to compare sdLDL-C to LDL-C between cases and controls. The association of diabetes with more extensive and severe CAD has been reported in angiographic studies ${ }^{28-33)}$, as well as in studies based on multislice coronary computed angiography ${ }^{34)}$. In addition, the major problem with PCI in diabetic patients is the high rate of restenosis, and these patients have a greater need for additional revascularization procedures because of late coronary atheroscle- 
Table 6. Serum lipid levels during the study period and the incidence of cardiovascular events among the 3 groups based on the presence or absence of lipid-lowering medication

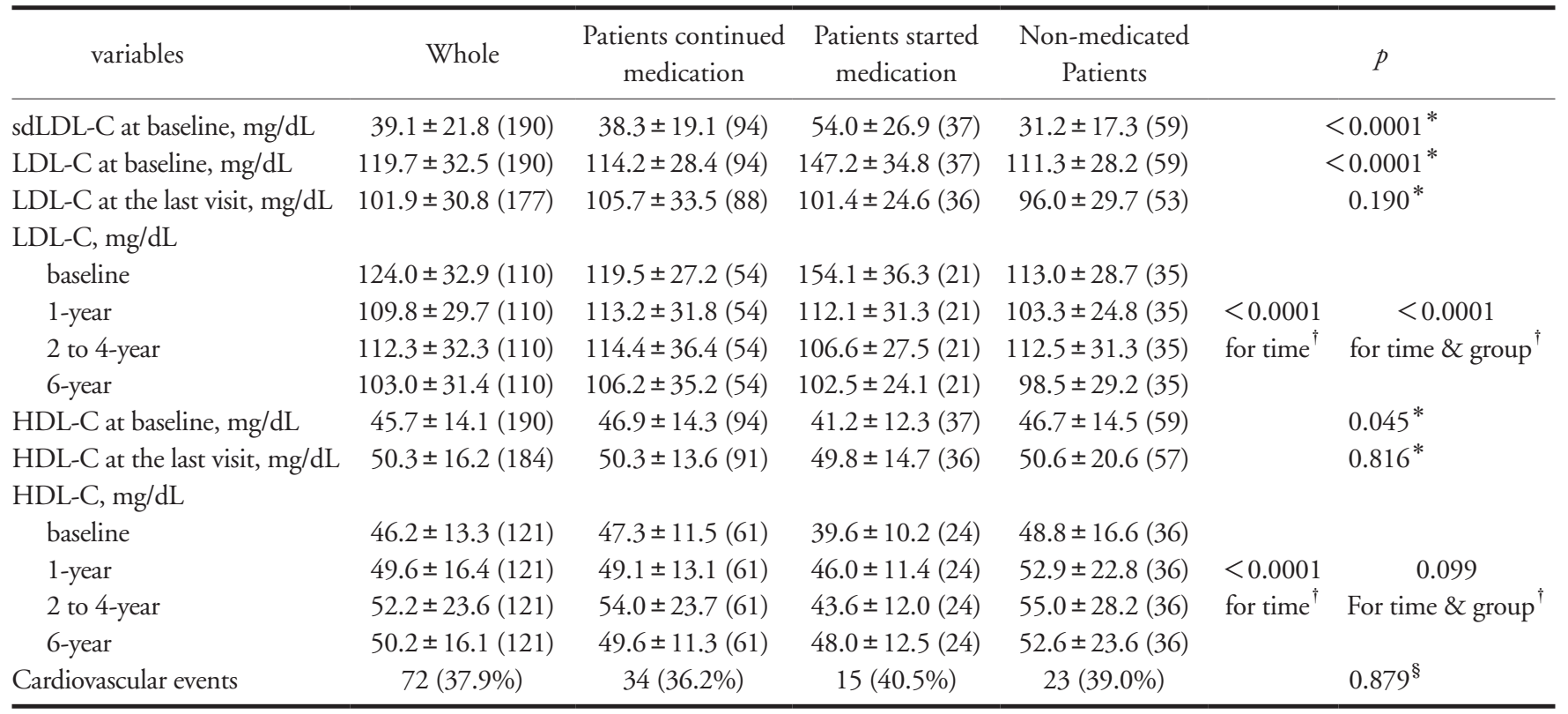

Data are expressed as the mean \pm SD (actual number of measured cases) or number (\%).

${ }^{*}$ the Wilcoxon test, ${ }^{\dagger}$ repeated-measures ANOVA, ${ }^{\S}$ the chi-square test

rosis progression ${ }^{28-31)}$. In the present study, cardiovascular events included any coronary revascularization. More than half of the patients required coronary revascularization at baseline. Therefore, it was accepted that patients with cardiovascular events were more likely to have undergone coronary revascularization procedure at baseline and more likely to have diabetes and be on insulin therapy; indeed, an increase in $\mathrm{HbA1c}$ was the leading risk factor for cardiovascular events in the present study. A previous study demonstrated that intensive insulin therapy decreased sdLDL-C measured by the same heparin magnesium precipitation method due to increased lipoprotein lipase levels and accelerated catabolism of triglyceriderich lipoproteins ${ }^{35)}$. Nevertheless, increases in the sdLDL-C/LDL-C ratio were significantly associated with cardiovascular events, as well as elevated HbA1c. To further explore this relationship, future studies with larger sample sizes are required to separately analyze data in diabetic and non-diabetic patients.

Numerous studies have reported that sdLDL is strongly associated with CVDs $\left.{ }^{2-4,} 13,23,26,27,36-41\right)$. A recent study investigating the association of cellular carotid plaque composition with cholesterol concentrations in lipoprotein sub-fractions evaluated by density gradient ultracentrifugation reported that the cholesterol content in the sdLDL fraction, but not the plasma LDL-C or HDL-C positively correlated with the macrophage-plaque content, while it negatively correlated with the smooth muscle cell content. This suggests that increased cholesterol in sdLDL is associated with unstable plaque ${ }^{38)}$. Previous studies compared LDL subclasses as evaluated by semi-quantitative estimation using gradient gel electrophoresis and angiographic changes in coronary arteries and demonstrated that an increase in sdLDL was strongly associated with coronary sclerosis progression. The Stanford Coronary Risk Intervention Project, a four-year, randomized, controlled clinical trial assessing the efficacy of multifactor risk reduction for reducing $\mathrm{CAD}$ progression, demonstrated that sdLDL was the single best lipoprotein predictor of increased stenosis ${ }^{39)}$. The Emory Angioplasty Versus Surgery Trial, a three-year, randomized, controlled clinical trial of PCI or CABG in patients with multivessel CAD, reported that sdLDL and hypertension were independently correlated with atherosclerosis progression in native coronary arteries ${ }^{40)}$. A sub-analysis of the HDL-Atherosclerosis Treatment study, a double-blind randomized controlled trial of simvastatin plus niacin and/or a mixture of antioxidants in patients with CAD and low HDL-C showed that on-study sdLDL concentrations were related to coronary atherosclerosis progression and cardiovascular events ${ }^{41)}$. The results of the present study are in line with the above-mentioned findings.

Previous meta-analyses of prospective epidemio- 
Table 7. Laboratory characteristics among the patients who did not take any lipid-lowering medication during follow-up and did not have a prior history of malignancy

\begin{tabular}{|c|c|c|c|c|}
\hline variables & $\begin{array}{l}\text { Whole } \\
(n=59)\end{array}$ & $\begin{array}{c}\text { Event } \\
(n=23)\end{array}$ & $\begin{array}{l}\text { Non-event } \\
\quad(n=36)\end{array}$ & $p$ \\
\hline LDL-C, mg/dL & $111.3 \pm 28.2$ & $107.3 \pm 27.1$ & $113.9 \pm 29.0$ & 0.794 \\
\hline sdLDL-C, mg/dL & $31.2 \pm 17.3$ & $36.0 \pm 22.9$ & $28.1 \pm 11.9$ & 0.082 \\
\hline sdLDL-C/LDL-C & $0.28 \pm 0.14$ & $0.33 \pm 0.18$ & $0.25 \pm 0.10$ & 0.055 \\
\hline LDL-PD, $\AA$ & $257.3 \pm 4.3$ & $257.1 \pm 4.9$ & $257.4 \pm 3.9$ & 0.441 \\
\hline sdLDL phenotype & $42.4 \%$ & $47.8 \%$ & $38.9 \%$ & 0.548 \\
\hline Large LDL-C, mg/dL & $80.2 \pm 25.0$ & $71.3 \pm 22.8$ & $85.8 \pm 25.0$ & 0.043 \\
\hline Triglycerides, mg/dL & $107.1 \pm 41.1$ & $107.0 \pm 36.9$ & $107.2 \pm 44.2$ & 0.859 \\
\hline HDL-C, mg/dL & $46.7 \pm 14.5$ & $45.0 \pm 19.6$ & $47.8 \pm 9.9$ & 0.072 \\
\hline Non HDL-C, mg/dL & $134.9 \pm 29.8$ & $134.1 \pm 26.7$ & $135.5 \pm 32.2$ & 0.921 \\
\hline LDL-C/HDL-C & $2.62 \pm 1.02$ & $2.75 \pm 1.08$ & $2.53 \pm 0.98$ & 0.123 \\
\hline Apolipoprotein A1, mg/dL & $119.6 \pm 23.7$ & $113.9 \pm 24.8$ & $123.3 \pm 22.6$ & 0.183 \\
\hline Apolipoprotein B, mg/dL & $85.8 \pm 17.7$ & $86.4 \pm 16.6$ & $85.4 \pm 18.7$ & 0.784 \\
\hline Lipoprotein(a), mg/dL & $18.4 \pm 18.8$ & $20.4 \pm 24.2$ & $17.1 \pm 14.6$ & 0.529 \\
\hline RLP-C, mg/dL & $4.5 \pm 2.0$ & $4.7 \pm 2.3$ & $4.4 \pm 1.8$ & 0.689 \\
\hline Glucose, mg/dL & $119.2 \pm 48.3$ & $125.7 \pm 43.5$ & $114.7 \pm 51.5$ & 0.074 \\
\hline HbA1c, \% & $6.15 \pm 1.03$ & $6.64 \pm 1.10$ & $5.84 \pm 0.86$ & 0.004 \\
\hline $\mathrm{eGFR}, \mathrm{mL} / \mathrm{min} / 1.73 \mathrm{~m}^{2}$ & $69.3 \pm 20.8$ & $61.1 \pm 21.3$ & $74.6 \pm 18.9$ & 0.121 \\
\hline hs-CRP, mg/dL & $0.53 \pm 1.67$ & $0.84 \pm 2.59$ & $0.32 \pm 0.55$ & 0.291 \\
\hline BNP, pg/mL & $151.5 \pm 263.3$ & $261.9 \pm 377.0$ & $76.7 \pm 90.8$ & 0.082 \\
\hline
\end{tabular}

Data are expressed as the mean $\pm \mathrm{SD}$, or $\%$.

logical and/or case-control studies and randomized controlled statin trials that compared LDL-C, nonHDL-C, and apolipoprotein B for the risk of cardiovascular events demonstrated that non-HDL-C and apolipoprotein B were somewhat superior to LDL$\mathrm{C}^{42-44)}$. Large prospective cohort studies using nuclear magnetic resonance spectroscopy to compare LDL particle number to LDL-C reported that LDL particle number was more strongly associated with the risk of cardiovascular events than LDL-C ${ }^{45-47)}$. These results support the hypothesis that the total number of atherogenic lipoproteins is more important than LDL-C for CVD risk. More atherogenic lipoproteins include RLPs, sdLDL, and lipoprotein $(\mathrm{a})^{48)}$. A recent large Danish cohort study investigating genotypes and nonfasting lipid levels for CAD risk demonstrated that a 1 $\mathrm{mmol} / \mathrm{L}(39 \mathrm{mg} / \mathrm{dL})$ increase in non-fasting RLP-C calculated as non-fasting total-cholesterol minus HDL-C minus LDL-C, was associated with a 2.8 -fold increased causal risk for $\mathrm{CAD}$, independent of reduced HDL-C level, whereas as $1 \mathrm{mmol} / \mathrm{L}$ increase in LDL-C was only associated with a 1.5 -fold causal risk ${ }^{49)}$. In addition, recent studies have shown that the LDL-C/ HDL-C ratio might be a positive predictor of progression and lipid-rich coronary plaque, which results in unstable coronary atherosclerotic lesion ${ }^{15,50)}$. The present study compared sdLDL-C to LDL-C, nonHDL-C, triglyceride, apolipoprotein B, lipoprotein(a), RLP-C, HDL-C, LDL-C/HDL-C ratio, and apolipoprotein $\mathrm{A} 1$ in the fasting state. Although sdLDL-C was positively associated with non-HDL-C, triglyceride, apolipoprotein B, RLP-C, and LDL-C/HDL-C and was negatively associated with HDL-C and apolipoprotein A1, only increased sdLDL-C and increased sdLDL-C/LDL-C ratios among apolipoprotein B-containing lipoproteins were significantly associated with cardiovascular events. In addition, among the patients who did not take any lipid-lowering agents during the study period and had relatively well-controlled dyslipidemia as determined by the LDL-C at their last visit (mean value of $96.0 \mathrm{mg} / \mathrm{dL}$ ), imbalance of cholesterolpoor sdLDL and cholesterol-rich large LDL might contribute to cardiovascular event occurrence. It is well known that sdLDL formation is closely associated with postprandial hyperglycemia and hyperlipidemia, both of which are also important risk factors for CVDs ${ }^{51,52)}$. Therefore, an increased sdLDL-C/LDL-C ratio in the fasting state might imply residual risk for cardiovascular events in CAD patients.

The present study compared various lipid and non-lipid biomarkers to predict future cardiovascular events. Coronary atherosclerosis severity as evaluated 
by Gensini score, elevated levels of BNP indicating advanced heart failure, the presence of diabetes and/or elevated $\mathrm{HbAlc}$, decreases in eGFR, inflammation reflected by CRP levels, and advanced age were stronger predictors for cardiovascular events than lipid markers. In addition, unlike BNP and eGFR, sdLDL$\mathrm{C}$ did not change with advanced age. Therefore, the significance of sdLDL-C level might be more pronounced in younger population, and future studies are needed to investigate these issues.

This study is associated with several other limitations. First, this was a very small cohort study, and we could not analyze the data separately by gender and/or by the presence or absence of diabetes. Second, cardiovascular events included coronary events related to unstable plaque, as well as revascularization for restenosis after PCI, any atherogenic cardiovascular diseases, and ischemic heart failure requiring hospitalization. Third, the effects of lipid-lowering therapy and target levels of sdLDL-C could not be investigated. Fourth, patients with cardiovascular events had more hypertension and diabetes; however, the magnitudes of blood pressure and glycemic controls were not evaluated. It is well known that atherosclerotic cardiovascular disease should be comprehensively managed. Future prospective studies should thus be conducted to evaluate these issues in larger sample sizes.

\section{Conclusion}

The present study of CAD patients demonstrated three findings. First, among LDL particles, sdLDL plays an important role in cardiovascular disease progression. Second, sdLDL-C is a very promising biomarker to predict future cardiovascular events. Third, the sdLDL-C/LDL-C ratio is an important residual risk in secondary prevention. A large cohort study is required to determine the appropriate target level of sdLDL-C.

\section{Acknowledgments}

We would like to thank the nursing stuff of the catheterization laboratory and all of the cardiologists at the Department of Cardiology of Showa University Hospital for their valuable help with this study. The present study was supported in part by a Showa University Grant-in-Aid for Innovative Collaborative Research Projects and a Special Research Grant-in-Aid for the Development of Characteristic Education from the Japanese Ministry of Education, Culture, Sports, Science, and Technology (Takashi Katagiri and Shinji Koba, 2003 and 2004).

\section{Disclosures}

Yasuki Ito is an employee of Denka Seiken. The other authors have no conflicts of interest to declare.

\section{References}

1) Sniderman A, Williams K, Cobbaert C: ApoB versus nonHDL-C: What to do when they disagree. Curr Atheroscler Rep, 2009; 11: 358-363

2) Berneis KK, Krauss RM: Metabolic origins and clinical significance of LDL heterogeneity. J Lipid Res, 2002; 43: 1363-1379

3) Brunzell JD: Increased apoB in small dense LDL particles predicts premature coronary artery disease. Arterioscler Thromb Vasc Biol, 2005; 25: 474-475

4) Griffin BA: Lipoprotein atherogenicity: an overview of current mechanisms. P Nutr Soc, 1999; 58: 163-169

5) Ensign W, Hill N, Heward CB: Disparate LDL phenotypic classification among 4 different methods assessing LDL particle characteristics. Clin Chem, 2006; 52: 17221727

6) Hirayama S, Miida T: Small dense LDL: An emerging risk factor for cardiovascular disease. Clin Chim Acta, 2012; 414: 215-224

7) Austin MA, Breslow JL, Hennekens CH, Buring JE, Willett WC, Krauss RM: Low-density lipoprotein subclass patterns and risk of myocardial infarction. JAMA, 1988; 260: 1917-1921

8) Koba S, Hirano T, Kondo T, Shibata M, Suzuki H, Murakami M, Geshi E, Katagiri T: Significance of small dense low-density lipoproteins and other risk factors in patients with various types of coronary heart diseases. Am Heart J, 2002; 144: 1026-35

9) St-Pierre AC, Cantin B, Dagenais GR, Mauriége P, Bernard PM, Després JP, Lamarche B: Low-density lipoprotein subfractions and the long-term risk of ischemic heart disease in men, 13-year follow-up data from the Québec cardiovascular study. Arterioscler Thromb Vasc Biol, 2005; 25: 553-559

10) Koba $S$, Hirano T, ItoY, Tsunoda, T, Yokota $Y$, Ban $Y$, Iso Y, Suzuki H, Katagiri T: Significance of small dense lowdensity lipoprotein-cholesterol concentrations in relation to the severity of coronary heart diseases. Atherosclerosis, 2006; 189: 206-214

11) Koba S, Yokota Y, Hirano T, Ito Y, Ban $Y$, Tsunoda T, Sato T, Shoji M, Suzuki H, Geshi E, Kobayashi Y, Katagiri T: Small LDL-cholesterol is superior to LDL-cholesterol in determining severe coronary atherosclerosis. J Atheroscler Thromb, 2008; 15: 250-260

12) Ai M, Otokozawa $S$, Asztalos BF, Ito $Y$, Nakajima $K$, White CC, Cupples LA, Wilson PW, Schaefer EJ: Small dense LDL cholesterol and coronary heart disease: results from the Framingham Offspring Study. Clin Chem, 2010; 56: 967-976

13) Arai H, Kokubo Y, Watanabe M, Sawamura T, Ito $Y$, Minagawa A, Okamura T, Miyamato Y: Small Dense LowDensity Lipoproteins Cholesterol can Predict Incident Cardiovascular Disease in an Urban Japanese Cohort: The 
Suita Study. J Atheroscler Thromb, 2013; 20: 195-203

14) Teramoto T, Sasaki J, Ishibashi $S$, Birou $S$, Daida H, Dohi S, Egusa G, Hiro T, Hirobe K, Iida M, Kihara S, Kinoshita M, Maruyama C, Ohta T, Okamura T, Yamashita S, Yokode M, Yokote K: Cardiovascular Disease Risk Factors Other than Dyslipidemia Executive Summary of the Japan Atherosclerosis Society (JAS) Guidelines for the Diagnosis and Prevention of Atherosclerotic Cardiovascular Diseases in Japan -2012 Version. J Atherscler Thromb, 2013; 20: 733-742

15) Nicholls SJ, Tuzcu EM, Sipahi I, Grasso AW, Schoenhagen P, Hu T, Wolski K, Crowe T, Desai MY, Hazen SL, Kapadia SR, Nissen SE: Statins, high-density lipoprotein cholesterol, and regression of coronary atherosclerosis. JAMA, 2007; 297: 499-508

16) Kashiwagi A, Kasuga M, Araki E, Oka Y, Hanafusa T, Ito $\mathrm{H}$, Tominaga M, Oikawa S, Noda M, Kawamura T, Sanke T, Namba M, Hashiramoto M, Sasahara T, Nishio Y, Kuwa K, Ueki K, Takei I, Umemoto M, Murakami M, Yamakado M, Yatomi Y, Ohashi H, Committee on the Standardization of Diabetes Mellitus-Related Laboratory Testing of Japan Diabetes Society (JDS): International clinical harmonization of glycated hemoglobin in Japan: From Japan Diabetes Society to National Glycohemoglobin Standardization Program values. Diabetol Int, 2012; 3: $8-10$

17) Teramoto T, Sasaki J, Ishibashi $S$, Birou $S$, Daida H, Dohi S, Egusa G, Hiro T, Hirobe K, Iida M, Kihara S, Kinoshita M, Maruyama C, Ohta T, Okamura T, Yamashita S, Yokode M, Yokote K: Executive Summary of the Japan Atherosclerosis Society (JAS) Guidelines for the Diagnosis and Prevention of Atherosclerotic Cardiovascular Diseases in Japan -2012 Version. J Atheroscler Thromb, 2013; 20: $517-523$

18) Imai $E$, Horio $M$, Watanabe $T$, Iseki $K$, Yamagata $K$, Hara S, Ura N, Kiyohara Y, Moriyama T, Ando Y, Fujimoto S, Konta T, Yokoyama H, Makino H, Hishida A, Matsuo S: Prevalence of chronic kidney disease in the Japanese general population. Clin Exp Nephrol, 2009; 13: 621-630

19) Gensini GG: A more meaningful scoring system for determining the severity of coronary heart disease. Am J Cardiol, 1983; 51: 606

20) Nakajima K, Saito K, Tamura A, Suzuki M, Nakano T, Adachi M, Tanaka A, Tada N, Nakamura H, Campos E, Havel RL: Cholesterol in remnant-like lipoproteins in human serum using monoclonal anti apoB-100 and anti apoA-1 immunoaffinity mixed gels. Clin Chim Acta, 1993; 223: 53-71

21) Okada M, Matsui H, Ito Y, Fujiwara A, Inano K: Lowdensity lipoprotein cholesterol can be chemically measured: a new superior method. J Lab Clin Med, 1998; 132: 195-201

22) Hirano T, Ito $Y$, Yoshino G: Measurement of small dense low-density lipoprotein particles. J Atheroscler Thromb, 2005; 12: 67-72

23) Hirano T, Ito Y, Saegusa H, Yoshino G: A novel and simple method for quantification of small dense LDL. J Lipid Res, 2003; 44: 2193-2201

24) Nichols AV, Krauss RM, Musliner TA: Nondenaturing polyacrylamide gradient gel electrophoresis. Methods
Enzymol, 1986; 128: 417-433

25) Roberts WL, Moulton L, Law TC, Farrow G, CooperAnderson M, Savory J, Rifai N: Evaluation of nine automated high-sensitivity C-reactive protein methods: Implications for clinical and epidemiological applications. Part 2. Clin Chem, 2001; 47: 418-425

26) Hirano T, Ito $Y$, Koba $S$, Toyoda M, Ikejiri A, Saegusa H, Yamazaki J, Yoshino G: Clinical significance of small dense low-density lipoprotein cholesterol levels determined by the simple precipitation method. Arterioscler Thromb Vasc Biology, 2004; 24: 558-563

27) Fukushima $Y$, Hirayama $S$, Ueno T, Dohi T, Miyazaki $T$, Ohmura H, Mokuno H, Miyauchi K, Miida T, Daida H: Small dense LDL cholesterol is a robust therapeutic marker of statin treatment in patients with acute coronary syndrome and metabolic syndrome. Clin Chim Acta, 2011; 412: 1423-1427

28) Stein B, Weintraub WS, Gebhart SSP, Cohen-Bernstein CL, Grosswald R, Liberman HA, Douglas Jr JS, Morris DC, King III SB: Influence of Diabetes Mellitus on Early and Late Outcome After Percutaneous Transluminal Coronary Angioplasty. Circulation, 1995; 91: 979-989

29) The Bypass Angioplasty Revascularization Investigation (BARI) Investigators: Comparison of coronary bypass surgery with angioplasty in patients with multivessel disease. N Engl J Med, 1996; 335: 217-225

30) Kip KE, Faxon DP, Detre KM, Yeh W, Kelsey SF, Currier JW; for the Investigators of the NHLBI PTCA Registry: Coronary Angioplasty in Diabetic Patients. The National Heart, Lung, and Blood Institute Percutaneous Transluminal Coronary Angioplasty Registry. Circulation, 1996; 94: 1818-1825

31) Norhammar A, Malmberg K, Diderholm E, Lagerqvist B, Lindahl B, Ryden L, Wallentin L: Diabetes mellitus: the major risk factor in unstable coronary artery disease even after consideration of the extent of coronary artery disease and benefits of revascularization. J Am Coll Cardiol, 2004; 43: 585-591

32) Ledru F, Ducimetiere P, Battaglia S, Courbon D, Beverelli F, Guize L, Guermonprez JL, Diebold B: New diagnostic criteria for diabetes and coronary artery disease: insights from an angiographic study. J Am Coll Cardiol, 2001; 37: 1543-1550

33) Niccoli G, Giubilato S, Di Vito L, Leo A, Cosentino N, Pitocco D, Marco V, Ghirlanda G, Prati F, Crea F: Severity of coronary atherosclerosis in patients with a first acute coronary event: a diabetes paradox. Eur Heart J, 2013; 34: 729-741

34) Ibebuogu UN, Nasir K, Gopal A, Ahmadi N, Mao SS, Young E, Honoris L, Nuguri VK, Lee RS, Usman N, Rostami B, Pal R, Flores F, Budoff MJ: Comparison of atherosclerotic plaque burden and composition between diabetic and non diabetic patients by non invasive CT angiography. Int J Cardiovasc Imaging, 2009; 25: 717-723

35) Hayashi $T$, Hirano $T$, Yamamoto $T$, Ito $Y$, Adachi $M$ : Intensive insulin therapy reduces small dense low-density lipoprotein particles in patients with type 2 diabetes mellitus: relationship to triglyceride-rich lipoprotein subspecies. Metabolism, 2006; 55: 879-884

36) Rizzo M, Pernice V, Frasheri A, Di Lorenzo G, Rini GB, 
Spinas GA, Berneis K: Small, dense low-density lipoproteins (LDL) are predictors of cardio- and cerebro-vascular events in subjects with the metabolic syndrome. Clin Endocrinol, 2009; 70: 870-875

37) Toft-Petersen AP, Tilsted HH, Aarøe J, Rasmussen K, Christensen T, Griffin BA, Aardestrup IV, Andreasen A, Schmidt EB: Small dense LDL particles--a predictor of coronary artery disease evaluated by invasive and CTbased techniques: a case-control study. Lipids Health Dis. 2011; 10: 21. doi: 10.1186/1476-511X-10-21

38) Zambon A, Puato M, Faggin E, Grego F, Rattazzi M, Pauletto P: Lipoprotein remnants and dense LDL are associated with features of unstable carotid plaque: a flag for non-HDL-C. Atherosclerosis, 2013; 230: 106-109

39) Williams PT, Superko HR, Haskell WL, Alderman EL, Blanche PJ, Holl LG, Krauss RM: Smallest LDL particles are most strongly related to coronary disease progression in men. Arterioscler Thromb Vasc Biol, 2003; 23: 314321

40) Zhao XQ, Kosinski AS, Barnhart HX, Superko HR, King SB 3rd: Prediction of native coronary artery disease progression following PTCA or CABG in the Emory Angioplasty Versus Surgery Trial. Med Sci Monit, 2003; 9: CR48-54

41) Williams PT, Zhao XQ, Marcovina SM, Brown BG, Krauss RM: Levels of cholesterol in small LDL particles predict atherosclerosis progression and incident CHD in the HDL-Atherosclerosis Treatment Study (HATS). PLoS One, 2013; 8: e56782

42) Emerging Risk Factors Collaboration, Di Angelantonio E, Sarwar N, Perry P, Kaptoge S, Ray KK, Thompson A, Wood AM, Lewington S, Sattar N, Packard CJ, Collins R, Thompson SG, Danesh J: Major lipids, apolipoproteins, and risk of vascular disease. JAMA, 2009; 302: 19932000

43) Sniderman AD, Williams K, Contois JH, Monroe HM, McQueen MJ, de Graaf J, Furberg CD: A meta-analysis of low-density lipoprotein cholesterol, non-high-density lipoprotein cholesterol, and apolipoprotein B as markers of cardiovascular risk. Circ Cardiovasc Qual Outcomes, 2011; 4: 337-345

44) Boekholdt SM, Arsenault BJ, Mora S, Pedersen TR, LaRosa JC, Nestel PJ, Simes RJ, Durrington P, Hitman GA, Welch KM, DeMicco DA, Zwinderman AH, Clearfield
MB, Downs JR, Tonkin AM, Colhoun HM, Gotto AM Jr, Ridker PM, Kastelein JJ: Association of LDL cholesterol, non-HDL cholesterol, and apolipoprotein B levels with risk of cardiovascular events among patients treated with statins: a meta-analysis. JAMA, 2012; 307: 13021309

45) Otvos JD, Mora S, Shalaurova I, Greenland P, Mackey RH, Goff DC Jr: Clinical implications of discordance between low-density lipoprotein cholesterol and particle number. J Clin Lipidol, 2011; 5: 105-113

46) Cromwell WC, Otvos JD, Keyes MJ, Pencina MJ, Sullivan L, Vasan RS, Wilson PW, D’Agostino RB: LDL Particle Number and Risk of Future Cardiovascular Disease in the Framingham Offspring Study - Implications for LDL Management. J Clin Lipidol, 2007; 1: 583-592

47) El Harchaoui K, van der Steeg WA, Stroes ES, Kuivenhoven JA, Otvos JD, Wareham NJ, Hutten BA, Kastelein JJ, Khaw KT, Boekholdt SM: Value of low-density lipoprotein particle number and size as predictors of coronary artery disease in apparently healthy men and women: the EPIC-Norfolk Prospective Population Study. J Am Coll Cardiol, 2007; 49: 547-553

48) Carmena R, Duriez P, Fruchart JC: Atherogenic lipoprotein particles in atherosclerosis. Circulation, 2004; 109 (Suppl 1): III2-III7

49) Varbo A, Benn M, Tybjærg-Hansen A, Jørgensen AB, Frikke-Schmidt R, Nordestgaard BG: Remnant cholesterol as a causal risk factor for ischemic heart disease. J Am Coll Cardiol, 2013; 61: 427-436

50) Kimura T, Itoh T, Fusazaki T, Matsui H, Sugawara S, Ogino Y, Endo H, Kobayashi K, Nakamura M: Low-density lipoprotein-cholesterol/high-density lipoprotein-cholesterol ratio predicts lipid-rich coronary plaque in patients with coronary artery disease. -Integrated-backscatter intravascular ultrasound study-. Circ J, 2010; 74: 1392-1398

51) Ban Y, Koba S, Tsunoda F, Yokota Y, Ezumi H, Kondo T, Suzuki H, Katagiri T: Predominance of small dense lowdensity lipoproteins and abnormal glucose regulation in patients with acute coronary syndrome. Circ J, 2006; 70: 393-401

52) Koba S, Tsunoda F, Hirano T, Iso Y, Suzuki H, Geshi E, Katagiri T: Postprandial changes in LDL phenotypes in patients with myocardial infarction. Eur J Clin Invest, 2005; 35: 171-179 